\title{
Poliquistosis renal. Un caso clínico y su aproximación a través de internet
}

\author{
J. F. Ávila de TOMÁs, J. M. PAJ ARes IZQUieRdo*
}

Médico de Familia. *Diplomado en enfermería. E.A.P. Humanes de Madrid.

Área 9 INSALUD. Madrid

\section{RESUMEN}

La poliquistosis renal $(P R)$ es la enfermedad ge nética más frecuente que afecta a más de 12,5 mi llones de personas de todo el mundo. De hecho im plica la aparición de múltiples quistes en cada riñón que crecen y se multiplican originando un aumento del tamaño renal. El riñón afectado clau dica originando una fase terminal de la enferme dad en la que la diálisis y el trasplante renal son las únicas alternativas terapéuticas.

Internet es una herramienta que permite obtener numerosa información veraz sobre la salud y la en fermedad. En este caso clínico se ha utilizado esta herramienta como fuente de información al paciente y de formación a los profesionales.

Palabras clave: Poliquistosis renal. hiperten sión arterial secundaria. Internet.
Polycystic kidney disease. A clinical case and its approximation throught internet

\begin{abstract}
Polycystic Kidney Disease is the most common genetic disease affecting more than 12.5 million people worldwide. In effect, polycystic kidney dise ase denotes multiple cysts on each kidney, which grow and multiply causing the kidney to increase. The diseased kidney shuts down causing end-stage renal disease for which dialysis and transplanta tion are the only forms of treatment.

Internet is a new tool that allows us to get truth ful information about health and disease. In this case report this tool has been used as an information source for the patient and formation source for sanitary staff.
\end{abstract}

Key words: Polycystic kidney disease. Secondary high blood pressure. Internet.

\section{CASO CLÍNICO}

Varón de 26 años de edad que acude a la consulta por presentar desde hace 10 días un dolor sordo en la región lumbar, no irradiado, de características inflamatorias. Entre sus antecedentes personales no se encuentran patologías crónicas de interés ni intervenciones quirúrgicas. Su madre ha sido trasplantada de riñón hace 6 años tras 7 años de hemodiálisis por una poliquistosis renal.

En la exploración se encuentra afebril, con una tensión arterial de 135/82 mmHg y se observa una contractura muscular a nivel lumbar con puñoper- cusión renal positiva. El resto de la exploración clínica es normal. En una tira de orina se objetiva hematuria. Con la sospecha clínica de cólico renoureteral, se inicia tratamiento con N-butilbromuro de hioscina $10 \mathrm{mg} / 8 \mathrm{~h}$ y diclofenaco $50 \mathrm{mg} / 8 \mathrm{~h}$ y se solicita radiografía de abdomen, bioquímica sanguínea, sistemático y sedimento de orina y orina de 24 horas con aclaramiento de creatinina.

Acude a las 72 horas por empeoramiento de los síntomas, con dolor más intenso que le despierta por la noche. En la exploración la puñopercusión renal es claramente positiva, se mantiene afebril y la tensión arterial es 140/92 $\mathrm{mmHg}$ que 
se relaciona con el dolor. Se solicita radiografía abdominal urgente que se informa como aumento de silueta renal bilateral con borramiento de las líneas del psoas. Se realiza ecografía abdominal objetivándose varios quistes de pared fina compatibles con poliquistosis renal del adulto. No se observan quistes en páncreas ni en hígado. Se realiza una urografía intravenosa informada como: riñones aumentados de tamaño con sistema intrarrenal distorsionado en relación con su patología de base (poliquistosis renal).

Se cambia el tratamiento por sulindal 200 $\mathrm{mg} / 12 \mathrm{~h}$ por persistencia del dolor con una mejoría significativa del mismo tras 15 días de tratamiento.

Los resultados analíticos son:

-Hemograma: hemoblobina $16,8 \mathrm{mg} / \mathrm{dl}$, hematocrito $49,5 \%$, volumen corpuscular medio (VCM) 89,7 fl, leucocitos $7490 / \mu 1$ con fórmula leucocitaria normal, plaquetas $306.000 / \mu 1$, velocidad de sedimentación $6 \mathrm{~mm} 1$ hora.

-Bioquímica plasmática: glucosa: $85 \mathrm{mg} / \mathrm{dl}$, creatinina $1,18 \mathrm{mg} / \mathrm{dl}$, urea $29 \mathrm{mg} / \mathrm{dl}$, úrico 8,5 $\mathrm{mg} / \mathrm{dl}$, colesterol total $171 \mathrm{mg} / \mathrm{dl}$, triglicéridos 99 mg/dl, AST 37 U/L ALT 71 U/L, GGT 22 U/L, sodio $140 \mathrm{mEq} / \mathrm{L}$, potasio $4,4 \mathrm{mEq} / \mathrm{L}$, calcio 9,6 $\mathrm{mEq} / \mathrm{L}$, fósforo $4,2 \mathrm{mEq} / \mathrm{L}$.

- Sistemático y sedimento de orina: normal salvo hematuria moderada. No se aprecia microalbuminuria ni proteinuria.

-Aclaramiento de creatinina: $88 \mathrm{ml} / \mathrm{min}$.

A los seis días de la última visita se encuentra sin dolor aunque persiste un malestar general inespecífico. La exploración está dentro de límites normales salvo una tensión arterial de $150 / 100 \mathrm{mmHg}$. que no es explicable por el dolor. Se deriva a consulta de enfermería para valoración inicial de hipertensión arterial secundaria a patología renal y se inicia tratamiento con enalapril $20 \mathrm{mg} / 24 \mathrm{~h}$ por el factor protector a medio plazo sobre la función renal ${ }^{1}$ y dieta hiposódica, con normalización de las cifras tensionales al octavo día del tratamiento.

Se realizó una valoración y seguimiento en la consulta de enfermería para el abordaje de diferentes aspectos de la enfermedad. La valoración inicial se realizó según los patrones funcionales de Gordon ${ }^{2}$.

-Conocimiento y control de la salud: el seguimiento de su madre, con patología similar, ha ayudado a que haya adquirido ciertos conocimientos y habilidades para su control. Aún así se aprecian importantes carencias y un conocimiento limitado de su patología.

-Nutricional-metabólico: hasta el momento seguía una dieta hipercalórica.

-Eliminación: en la eliminación urinaria no se observan cambios significativos. La elimina- ción fecal es normal y diaria.

-Actividad-ejercicio: el paciente no realiza ningún tipo de ejercicio.

-Sueño-descanso: en algunos periodos, por la sintomatología (dolor), existe una disminución de la calidad del sueño con frecuentes interrupciones del mismo.

-Cognoscitivo-perceptivo: presencia de dolores en región lumbar.

-Percepción de sí mismo: progresiva pérdida de autoestima y un ligero aumento de la ansiedad.

Las sucesivas consultas de enfermería proporcionaron los siguientes diagnósticos:

-Déficit de conocimientos relacionados con falta de información sobre su patología y cuidados.

-Dolor relacionado con presión en órganos y tejidos abdominales caracterizado por el aumento del tamaño de los quistes.

- Ansiedad y afrontamiento ineficaz relacionado con el diagnóstico, tratamiento y pronóstico.

\section{DISCUSIÓN}

La poliquistosis renal autosómica dominante, también denominada poliquistosis renal del adulto, es una de las enfermedades genéticas más comunes (aproximadamente 1 de cada 1.000 individuos está afectado). La característica más llamativa de esta enfermedad es el desarrollo de quistes en los riñones que conducen, generalmente, a un fallo renal en la vida adulta ${ }^{3}$. Las manifestaciones extrarrenales incluyen quistes en el hígado y, más raramente, quistes en el páncreas y otros órganos ${ }^{4}$. Los aneurismas intracraneales ocurren en aproximadamente el 5\% de los pacientes y representan una causa significativa de morbilidad y mortalidad debida a hemorragia subaracnoidea $^{5}$. Sin embargo, la principal causa de morbilidad es la enfermedad renal progresiva caracterizada por la formación y aumento de tamaño de quistes rellenos de fluido. Debido a esto, la función renal se deteriora y conduce a una enfermedad terminal en más del $50 \%$ de los pacientes a la edad de 60 años ${ }^{6}$.

Los objetivos que nos planteamos desde un inicio fueron: informar al paciente sobre su proceso e intentar establecer unos niveles adecuados de autocuidados basándonos en sus posibilidades, reducir al máximo posible los episodios de dolor, reducir a niveles aceptables las cifras de tensión arterial, aumentar su autoestima a niveles previos al diagnóstico y reducir al máximo la ansiedad.

El caso clínico descrito tiene una característica diferenciadora ya que se ha introducido un nuevo elemento que ha venido a completar los múltiples 
DIRECCIONES DE INTERNET UTILIZADAS PARA LA OBTENCIÓN DE INFORMACIÓN EN ESTE CASO CLÍNICO

Renal - El portal de nefrología en español

Información de la Sociedad Española de Nefrología

The Polycystic Kidney Research Foundation Home Page.

Hypertension, Dialysis and Clinical Nephrology

National Kidney Foundation - Making Lives Better

International Society of Nephrology.

United States Renal Data System (USRDS)

Sociedad española de nefrología

Renalnet

Sociedad Latinoamericana de Nefrología e Hipertensión

Arizona Dietetic project

\author{
http:/ / www.renal.com.ar/ \\ http:/ / www.senefro.org/ \\ http:/ / www.pkdcure.org/ \\ http:/ / www.hden.com/ \\ http:/ / www.kidney.org/ \\ http:/ / www.isn-online.org/ \\ http:/ / www.usrds.org/ \\ http:/ / www.senefro.org/ \\ http:// www.renalnet.org/ \\ http:/ / www.slanh.org/ \\ http:/ / www.nephron.com/adp/
}

interrogantes surgidos desde la primera consulta. Este elemento clave ha sido el uso de Internet como una herramienta más que nos ha mostrado desde los últimos avances en el tratamiento de la poliquistosis renal hasta detalles sobre los grupos de autoayuda más cercanos al lugar de residencia del paciente. Toda la información que se ha utilizado ya existía en otros soportes pero Internet la ha unificado y hecho más accesible.

Se realizaron múltiples consultas a través de la Red. Por un lado se accedió a Medline®, a través de PubMed en http://www.ncbi.nlm.nih.gov. Se estableció una búsqueda mediante las palabras claves "polycystic kidney disease" limitándola a ensayos clínicos o revisiones. Se realizó una segunda búsqueda de guías clínicas mediante la sentencia de búsqueda "progressive chronic renal failure".

La segunda incursión en Internet fue a través del buscador Google ${ }^{\circledR}$, en http://www.google.com con la intención de localizar información existente en Internet no procedente de literatura científica pero que se ajustara a unos mínimos criterios de calidad de información. En este buscador se encontraron 271 referencias en español en la búsqueda "poliquistosis renal" y más de 16.600 en la búsqueda de "polycystic kidney disease". De ellas descartamos las que no procedían de fuentes sanitarias reconocidas como páginas personales, noticias de revistas o periódicos y páginas cuya fuente no estaba aclarada o no existía constancia de la responsabilidad de los contenidos expuestos; de forma que disminuimos la búsqueda a un total de 6.507 páginas. Como su número continuaba siendo muy alto seleccionamos a través del propio buscador, mediante la opción de búsqueda de páginas relacionadas, las páginas procedentes de sociedades científicas o asociaciones de afectados resultando un total de 720 páginas que correspondían a 65 sitios web. Una vez consultadas todas, escogimos las que se sometían a alguno de los criterios de calidad dentro de la red (HONcode o Internet Health Coalition) y que ofrecían mayor información sobre la poliquistosis renal. De esta forma minimizamos la consulta a $11 \mathrm{si-}$ tios que se exponen en la tabla I.

De cada una de estas páginas obtuvimos información importante para realizar una correcta labor de asesoramiento e información del paciente hasta su consulta con el servicio de nefrología. La clasificación de los datos obtenidos en la búsqueda la estructuramos en dos apartados: de importancia para los profesionales sanitarios y de importancia para el paciente. En el primero de los grupos incluimos información que contenía datos clínicos y pronósticos. En el segundo de ellos agrupamos datos referentes a información general de la enfermedad y a hábitos de vida encaminados a proteger la función renal (información sobre dieta, fármacos y estilos de vida).

Encontramos numerosa información sobre dietas de protección renal en Clinical Practice Guidelines for Nutrition in Chronic Renal Failure de la Natio nal Kidney Fundation en http://www. kidney. org/professionals/doqi/doqi/doqi_nut.html, y en The Arizona Dietetic project en http://www. nephron.com/adp/, que además tiene traducción al español. Una buena fuente de datos de información tanto para el paciente como para el profesional sanitario la encontramos en el "Early renal guidebo $o k$ " (http://nephron.com/fkgframeset.html), una pequeña guía clínica multimedia que explica diferentes aspectos sobre la fisiología del riñón y la fisiopatología de la insuficiencia renal. 
Una tercera aproximación fue a través de las revistas American Journal of Kidney Diseases en http://ajkd.highwire.org/ y Nutrition de la Sociedad Americana de Nutrición en www.nutrition.org. En la primera dirección pudimos acceder a numerosos artículos a texto completo referentes a ensayos clínicos, guías de consenso o revisiones ${ }^{7-9}$, y en la segunda a ensayos clínicos referentes a dietas de protección de insuficiencia renal en animales ${ }^{10-12}$.

\section{CORRESPONDENCIA:}

José Francisco Ávila de Tomás

EAP Humanes de Madrid

C/ Ferrocarril, 4

28970 Humanes de Madrid. Madrid

E-mail: joseavil@eresmas.com

\section{Bibliografía}

1. Ecder T, Chapman AB, Brosnahan GM, Edelstein CL, Johnson AM, Schrier RW. Effect of antihypertensive therapy on renal function and urinary albumin excretion in hypertensive patients with autosomal dominant polycystic kidney disease. Am J Kidney Dis 2000; 35(3): 427-32.

2. Gordon M. Nursing Diagnosis: Process and application. New York: McGraw Hill, 1982

3. Torra R, Badenas C, Darnell A, Nicolau C, Volpini V, Revert L, et al. Estudio clínico, genético y molecular de la poliquistosis renal autosómica dominante tipos 1 y 2 . Med Clin (Barc) 1998; 110:481-7.

4. Gabow PA. Autosomal dominant polycystic kidney disease. N Eng J Med, 1993, 329:32.

5. Parfrey PS, Bear JC, Morgan J, Cramer BC, McManamon PJ, Gault MH. The diagnosis and prognosis of autosomal dominant polycystic kidney disease. N Engl J Med 1990; 323: 1085-90.

6. Asplin JR, Coe FL. Enfermedades tubulares hereditarias. En: Fauci AS, Braunwald E, Isselbacher K, Wilson J, Mar- tín J, Kasper D, et al. Harrison Principios de Medicina Interna. 14 ed. Madrid: Ed McGraw Hill, 1998.

7. JG Porush. Hypertension and chronic renal failure: the use of ACE inhibitors. Am J Kidney Dis 1998, 31: 177-84.

8. Mackenzie HS, Brenner BM. Current strategies for retarding progression of renal disease Am J Kidney Dis 1998, 31: 161-70.

9. Cotton CU, Avner ED, PKD and CF: an interesting family provides insight into the molecular pathophysiology of polycystic kidney disease Am J Kidney Dis 1998; 32: 1081-3.

10. Kopple JD. Pathophysiology of Protein-Energy Wasting in Chronic Renal Failure J Nutr. 1999; 129: 247S-51S.

11. Jayapalan S, Saboorian MH, Edmunds JW, Aukema HM. High Dietary Fat Intake Increases Renal Cyst Disease Progression in Han: SPRD-cy Rats J Nutr. 2000 130: 2356-60.

12. Stern JS, Gades MD, Wheeldon CM, Borchers AT. Calorie Restriction in Obesity: Prevention of Kidney Disease J Nutr 2001; 131: 913S-7S. 